\title{
Optical and infrared properties of V1647 Orionis during the 2003-2006 outburst
}

\section{Temporal evolution of the eruptive source}

\author{
D. Fedele ${ }^{1,2}$, M. E. van den Ancker ${ }^{1}$, M. G. Petr-Gotzens ${ }^{1}$, and P. Rafanelli ${ }^{2, \star}$ \\ ${ }^{1}$ European Southern Observatory, Karl Schwarzschild Strasse 2, 85748 Garching bei München, Germany \\ e-mail: dfedele@eso.org \\ 2 Dipartimento di Astronomia, Università degli studi di Padova, Vicolo dell’Osservatorio 2, 35122 Padova, Italy
}

Received 26 April 2007 / Accepted 4 June 2007

\begin{abstract}
Aims. The occurrence of new FU Orionis-like objects is fundamental to understand the outburst mechanism in young stars and their role in star formation and disk evolution. Our work is aimed at investigating the properties of the recent outburst of V1647 Ori.

Methods. Using optical and mid infrared long slit spectroscopy we monitored V1647 Orionis in outburst between February 2004 and January 2006.

Results. The optical spectrum is characterized by $\mathrm{H} \alpha$ and $\mathrm{H} \beta$ in P-Cygni profile and by many weak Fe I and Fe II emission lines. Short timescale variability was measured in the continuum and line emission. In January 2006 we detected for the first time forbidden emission lines ([O I], [S II] and [Fe II]). These lines are likely produced by a Herbig-Haro object driven by V1647 Ori. The mid infrared spectrum of V1647 Ori is flat and featureless at all epochs. The SED changed drastically: the source was much redder in the early outburst than in the final phase. The magnitude rise and the SED of V1647 Ori resembles that of a FUor while the duration and recurrence of the outburst resemble that of a EXor. The optical spectrum is clearly distinct from either the absorption line spectrum of a FUor or the T Tauri-like spectrum of an EXor.

Conclusions. Our data are consistent with a disk instability event which led to an increase of the mass accretion rate. The data also suggest the presence of a circumstellar envelope around the star+disk system. The peculiar $N$ band spectrum might be explained by dust sublimation in the outer layers of the disk. The presence of the envelope and the outburst statistics suggest that these instability events occur only in a specific stage of a Class I source (e.g. in the transition phase to an optically visible star surrounded by a protoplanetary disk). We discuss the outburst mechanisms in term of the thermal instability model.
\end{abstract}

Key words. stars: planetary systems: protoplanetary disks - accretion, accretion disks - instabilities - ISM: Herbig-Haro objects

\section{Introduction}

One of the clearest pieces of evidence for disk accretion during early stages of stellar evolution are events like FU Orionis and EX Lupi outbursts (Herbig 1977; Hartmann \& Kenyon 1996). These outbursts are thought to be the consequence of a sudden and steep increase of the mass accretion rate onto the central star, which changes from those commonly found around T Tauri stars $\left(\sim 10^{-7} M_{\odot} \mathrm{yr}^{-1}\right)$ into values of $10^{-3}-10^{-4} M_{\odot} \mathrm{yr}^{-1}$. Statistical studies suggest that young low-mass stars experience several FU Orionis-like outbursts during the early phase of stellar evolution. The emergence of a new pre-main-sequence outburst object is thus a unique opportunity to address the physical processes that occur in the disk's interior. For this reason, several astronomers have focused their attention on the recent outburst of V1647 Orionis.

V1647 Ori is a young eruptive star known to be the illuminating source of McNeil's Nebula, a reflecting nebula discovered by the amateur astronomer J.W. McNeil in January 2004 (McNeil et al. 2004). In the months following the discovery,

^ Based on observations collected at the European Southern Observatory, Paranal, Chile. Proposal ID: 272.C-5045, 074.C-0679, 276.C-5022. the star, located in the L1630 cloud within the Orion B molecular cloud complex, showed an increase of its optical/IR brightness of up to $6 \mathrm{mag}$. The outburst has been observed from the X-ray regime (e.g. Grosso et al. 2005) to infrared wavelengths (see e.g. Muzerolle et al. 2005; Andrews et al. 2004). In February 2004, 4 months after the onset of the outburst, the brightness rise stopped and the magnitude remained (relatively) constant. In November 2005, a communication by Kospal et al. (2005) claimed the beginning of a fast fading phase in the optical light of V1647 Ori. The system is further characterized by a red energy distribution and by many emission lines in its optical and near-IR spectrum. Apart from the Brackett series (seen purely in emission), all the Hydrogen lines exhibit a P-Cygni profile, which indicates mass outflow in a wind. Vacca et al. (2004) find that their near-IR emission line spectrum is consistent with a dense and ionized wind model where the optically thick $\mathrm{H}$ lines are produced. The same model is able to explain why the outburst has not been seen at radio wavelengths (Andrews et al. 2004). V1647 Ori is known to have experienced a previous outburst, as is clear from the appearance of the reflection nebula in 1996-1997 in the atlas of Mallas \& Kreimer (1970) and as recently confirmed by Aspin et al. (2006). Furthermore, its optical and near-IR spectrum does not resemble any other 
Table 1. Log of spectroscopic observations with FORS2 of V1647 Ori.

\begin{tabular}{|c|c|c|c|c|c|c|c|c|}
\hline $\begin{array}{l}\text { Date } \\
\text { (UT) }\end{array}$ & "JD -2 450000 & $\begin{array}{c}F W H M \\
(")\end{array}$ & $\begin{array}{c}\text { Slit width } \\
\left({ }^{\prime \prime}\right)\end{array}$ & $\begin{array}{c}\text { Slit PA } \\
\left({ }^{\circ}\right)\end{array}$ & $\begin{array}{c}\text { Spectral range } \\
(\AA)\end{array}$ & $\begin{array}{c}\text { Exposure time } \\
\text { (s) }\end{array}$ & 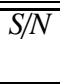 & $\begin{array}{c}R_{\mathrm{C}} \\
(\mathrm{mag})\end{array}$ \\
\hline 2004-02-18 & 3053.081 & 0.85 & 1.0 & 45.0 & $4560-5860$ & 700 & 20 & $17.39 \pm 0.10$ \\
\hline $2004-02-23$ & 3058.090 & 1.25 & 1.0 & 45.0 & $4560-5860$ & 700 & 19 & $17.31 \pm 0.10$ \\
\hline 2004-03-13 & 3077.018 & 0.9 & 1.0 & 45.0 & $4560-5860$ & 700 & 14 & $17.52 \pm 0.10$ \\
\hline 2004-03-18 & 3082.011 & 1.15 & 1.0 & 45.0 & $4560-5860$ & 700 & 13 & $17.42 \pm 0.10$ \\
\hline 2004-03-27 & 3091.020 & 0.9 & 1.0 & 45.0 & $4560-5860$ & 700 & 14 & $17.31 \pm 0.10$ \\
\hline 2004-12-08 & 3347.315 & 1.2 & 0.7 & 90.0 & $4560-5860$ & 500 & 9 & $17.23 \pm 0.05$ \\
\hline $2004-12-21$ & 3360.251 & 0.65 & 0.7 & 90.0 & $4560-5860$ & 500 & 14 & $16.87 \pm 0.05$ \\
\hline 2005-01-05 & 3375.180 & 1.35 & 0.7 & 90.0 & $4560-5860$ & 500 & 10 & $17.07 \pm 0.05$ \\
\hline $2005-02-18$ & 3419.126 & 1.0 & 0.7 & 90.0 & $4560-5860$ & 500 & 4 & $17.77 \pm 0.05$ \\
\hline $2005-02-29$ & 3430.114 & 0.65 & 0.7 & 90.0 & $4560-5860$ & 500 & 9 & $17.12 \pm 0.05$ \\
\hline $2005-03-15$ & 3444.045 & 0.72 & 0.7 & 90.0 & $4560-5860$ & 500 & 7 & $17.50 \pm 0.05$ \\
\hline $2005-12-27$ & 3731.215 & 1.33 & 0.7 & 90.0 & $4560-5860$ & $4 \times 1800$ & 2 & $20.74 \pm 0.11$ \\
\hline $2004-12-08$ & 3347.322 & 1.15 & 0.7 & 90.0 & $5750-7310$ & 500 & 20 & $17.23 \pm 0.05$ \\
\hline $2004-12-21$ & 3360.258 & 0.68 & 0.7 & 90.0 & $5750-7310$ & 500 & 42 & $16.87 \pm 0.05$ \\
\hline 2005-01-05 & 3375.187 & 1.08 & 0.7 & 90.0 & $5750-7310$ & 500 & 26 & $17.07 \pm 0.05$ \\
\hline $2005-02-18$ & 3419.133 & 1.12 & 0.7 & 90.0 & $5750-7310$ & 500 & 15 & $17.77 \pm 0.05$ \\
\hline $2005-02-29$ & 3430.121 & 0.62 & 0.7 & 90.0 & $5750-7310$ & 500 & 23 & $17.12 \pm 0.05$ \\
\hline $2005-03-15$ & 3444.052 & 0.69 & 0.7 & 90.0 & $5750-7310$ & 500 & 22 & $17.50 \pm 0.05$ \\
\hline 2006-01-29 & 3764.122 & 0.73 & 0.7 & 90.0 & $5750-7310$ & $4 \times 1800$ & 4 & $22.05 \pm 0.11$ \\
\hline
\end{tabular}

previous spectra of FUors or EXors objects (Reiburth \& Aspin 2004; Vacca et al. 2004). The 2-3 year duration of the outburst, its recurrence on a timescale of decades and the "peculiar" spectrum of V1647 Ori, are important clues for the comprehension of outburst events in pre-main-sequence stars.

From February 2004 to January 2006 we have followed the evolution of the outburst of V1647 Ori at optical (4700-7300 $\AA$ ) and mid-IR $(8-13 \mu \mathrm{m})$ wavelengths. Here, we present the results of our photometric and spectroscopic monitoring of the eruptive source. The analysis of the reflection nebula is presented in a separate paper (Fedele et al. 2007, hereafter Paper I). Observations and data reduction are described in Sect. 2. In Sect. 3 we analyze the observations. A discussion of the results is presented in Sect. 4. We draw the conclusions in Sect. 5.

\section{Observations and data reduction}

Observations were performed using FORS2 at ESO's Very Large Telescope in Paranal, Chile and TIMMI2 at the $3.6 \mathrm{~m}$ telescope at La Silla. FORS2 (Appenzeller et al. 1998) is an optical facility (3000-10000 §) which allows imaging in different bands and grism spectroscopy. TIMMI2 (Käufl et al. 2003) is a mid-infrared $(8-14 \mu \mathrm{m})$ multi-mode instrument including a lowand medium-resolution spectrograph. We have also included in our analysis some publicly available VLTI/MIDI observations of V1647 Ori taken from the ESO archive $^{1}$. MIDI is the midinfrared beam-combiner facility of the ESO VLT interferometer (Leinert et al. 2003).

\subsection{Optical spectroscopy}

12 long slit spectra of V1647 Ori were obtained with FORS2 and with the grism $1400 \mathrm{~V}(4560-5860 \AA, \lambda / \Delta \lambda \sim 2100)$ between 2004 February 18 and 2005 December 27. Seven further long slit spectra were obtained between 2004 December 08 and 2006 January 29 , with the grism $1200 \mathrm{R}(5750-7310 \AA, \lambda / \Delta \lambda \sim$ 2100). A detailed log of the observations is reported in Table 1.

\footnotetext{
${ }^{1}$ http://www.eso.org/archive
}

A standard optical long slit spectrum extraction procedure was applied to reduce the raw data - bias subtractions, flatfielding, wavelength calibration, cosmic rays and sky background removal and weighted average along the spatial axis. Observations of spectro-photometric standard stars during each night allowed us to compute the sensitivity function of the spectrograph. To flux-calibrate the spectra of V1647 Ori, the 1-dimensional extracted spectra were first multiplied by the sensitivity function - to compute the exact slope of the spectrum and then scaled to the flux level measured from the acquisition images. This is straightforward for the red spectra since the acquisition images were taken with the same filter. For the blue spectra we have assumed $\left(V-R_{\mathrm{C}}\right) \sim 1.8 \pm 0.2$ (as measured by McGehee et al. 2004 in February-April 2004 and from Kospal et al. 2005 in October 2005) from which we computed the absolute $V$ flux level.

Differential aperture photometry of V1647 Ori has been computed over an aperture radius of 2 '.52 (10 pixels) from the acquisition images $\left(R_{\mathrm{C}}\right.$ filter). In Paper I we computed the $R_{\mathrm{C}}$ magnitude of two reference stars in the FORS2 frames which were used to calibrate the instrumental magnitude of the acquisition images discussed here. The two stars were found to be not variable and to have the following $R_{\mathrm{C}}$ magnitude: $\mathrm{RA}(\mathrm{J} 2000)=$ 05:46:09.71; $\operatorname{Dec}(\mathrm{J} 2000)=-00: 03: 31.1 ; R_{\mathrm{C}}=20.08 \pm 0.09$ and $\mathrm{RA}(\mathrm{J} 2000)=05: 46: 05.88 ; \operatorname{Dec}(\mathrm{J} 2000)=-00: 02: 39.7 ; R_{\mathrm{C}}=$ $16.39 \pm 0.02$. The latter corresponds to the comparison star "E" in Semkov (2004) and (2006), who measured $R_{\mathrm{C}}=16.39 \pm 0.02$. The results are listed in Table 1.

\subsection{Mid-IR spectroscopy}

\subsubsection{TIMMI2}

Low-resolution $(\lambda / \Delta \lambda \approx 200) N^{\prime}$-band $(7.7-13.0 \mu \mathrm{m})$ spectra of V1647 Ori (IRAS 05436-0007) were obtained on 2004 March 08 (JD 2453 072.526) and 2006 January 10 (JD 2453746.663 ) using the TIMMI2 instrument on the ESO $3.6 \mathrm{~m}$ telescope at La Silla. Sky subtraction was achieved by chopping in the North direction with an amplitude of $10^{\prime \prime}$ followed by a nodding pattern with the opposite direction and amplitude. The total integration time was $25 \mathrm{~min}$ per spectrum. The 


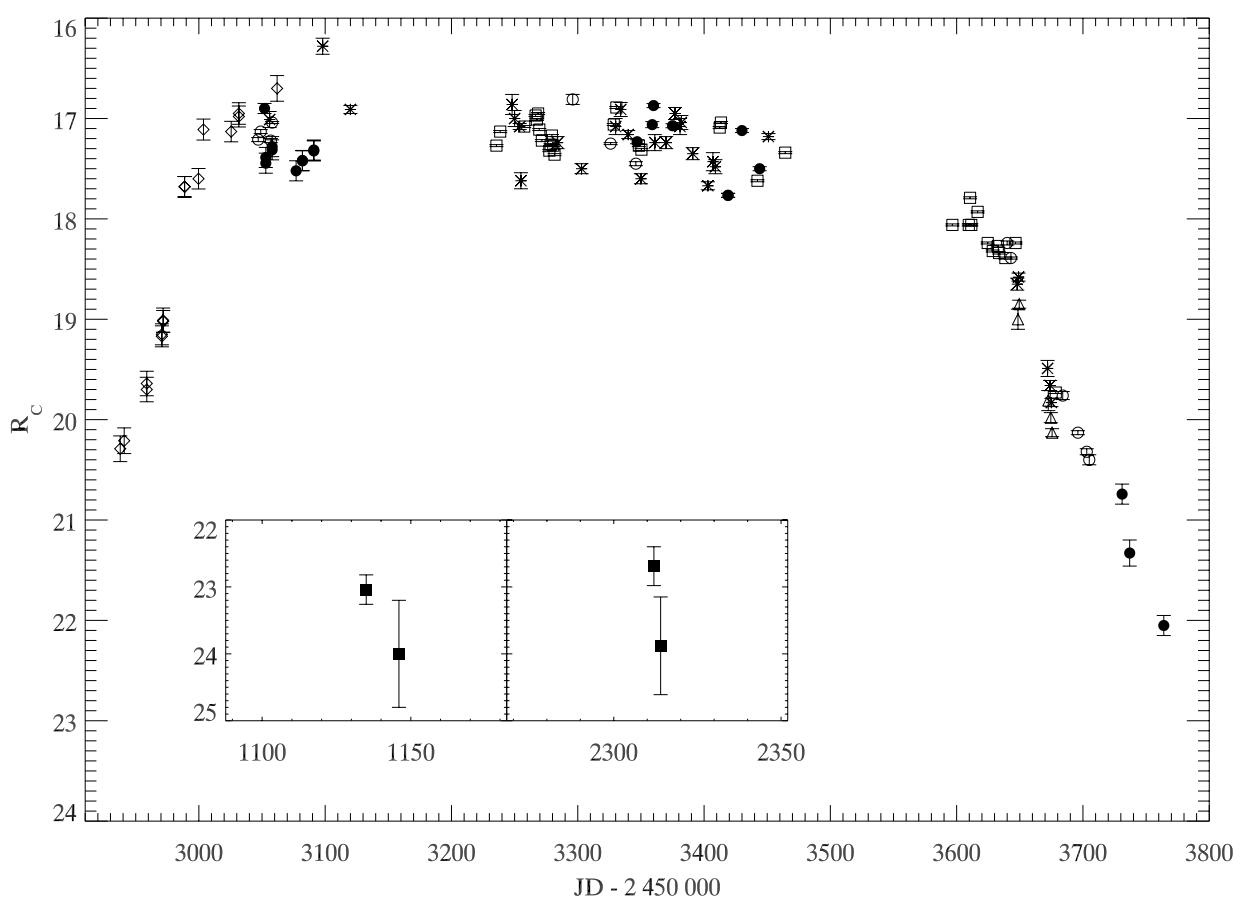

Fig. 1. Optical, $R_{\mathrm{C}}$, light curve of V1647 Ori. Data are from: filled circles this work; squares Semkov, E.H. (2006, 2004); triangles Kospal et al. (2005); stars Acosta-Pulido et al. (2007); diamonds Briceño et al. (2004); filled squares McGehee et al. (2004); open circles Ojha et al. (2006); star Acosta-Pulido et al. (2007). An offset of $-0.3 \mathrm{mag}$ was applied to the imported data (apart from those of Acosta-Pulido et al. 2007) in order to reach a similar magnitude level as our data. In the inset the pre-outburst magnitude level reported by McGehee et al. (2004) is shown.

slit, with a width of 1 .' 2 , was centered on a compact source detected in the $N^{\prime}$-band acquisition image. A spectrum of the reference star HD 37160 (K0III) was obtained before or after each IRAS 05436-0007 observation. Data were reduced using the usual steps of residual background subtraction, spectral extraction, and wavelength calibration. Correction for the telluric ozone absorption bands, as well as absolute flux calibration, were achieved by ratio-ing the IRAS 05436-0007 spectrum to that of HD 37160, flux-calibrated using the spectral templates by Cohen et al. (Cohen et al. 1999). The resulting spectra are shown in Fig. 6.

\subsubsection{VLTI/MIDI}

We also analyzed interferometric observations of V1647 Ori obtained with VLTI/MIDI on 3 nights between 2004 December 30 and 2005 March 01. The interferometric data were previously analyzed by Abraham et al. (2006). Hence we will discuss here only the MIDI low-resolution $(R=30)$ spectrum acquired on each night by the instrument after the interferometric observation.

Using the MIA+EWS-1.3 $3^{2}$ software package, a fixed mask was applied to the MIDI chopped spectrum. The background was estimated from the off-source (sky) frames and then subtracted from the on-source frames. The one-dimensional spectrum was extracted. In the same way, spectra of MIDI calibrator stars were extracted.

Aperture photometry was computed from the acquisition images with a narrow filter centered at $8.7 \mu \mathrm{m}$ adopting a fixed aperture of 12 pixel $\left(1{ }^{\prime \prime} 0\right)$. The throughput of the two MIDI channels are, for an unknown reason, different, and the aperture photometry differs for the two telescopes. We used the results

\footnotetext{
${ }^{2}$ http://WWw.strw.leidenuniv.nl/ koehler/MIA+EWSManual
}

from channel $\mathrm{B}$ which tend to be the more stable of the two. Conversion factors from counts to Jy were computed from the MIDI calibrators, whose $8.7 \mu \mathrm{m}$ flux were evaluated from theoretical spectral energy distributions. Energy distributions were derived from the Cohen list (Cohen et al. 1999) of infrared standard stars for the calibrator HD $37160\left(F_{8.7 \mu \mathrm{m}}=11.5 \mathrm{Jy}\right)$, and by matching the spectral type of the calibrator to stars in the Cohen list and scaling the spectrum by the ratio of their IRAS $12 \mathrm{mi}$ cron fluxes for HD $107446\left(F_{8.7 \mu \mathrm{m}}=37.9 \mathrm{Jy}\right)$ and HD 50778 $\left(F_{8.7 \mu \mathrm{m}}=28.9 \mathrm{Jy}\right)$. The three calibrators were also used to compute the sensitivity function of MIDI (wavelength dependence of the instrument's response). The spectrum of V1647 Ori has been flux calibrated by multiplying it by the sensitivity function and by scaling it in order to match the $8.7 \mu \mathrm{m}$ flux. The result is shown in Fig. 6.

\section{Results}

\subsection{Optical lightcurve}

Figure 1 shows the light curve of V1647 Ori in the $R_{\mathrm{C}}$ band based on the data of Table 1 and on previous measurements by other authors. Due to the influence of McNeil's nebula on the computation of the stellar flux and subtraction of local background, measurements with different instruments may result in a different magnitude estimation. In particular, given the better spatial resolution of our data, we may better disentangle the contribution from V1647 Ori from that of the nebula. For this reason, an offset of -0.3 mag was applied to $R_{\mathrm{C}}$ measurements by other authors. Such an offset is not needed for the data of Acosta-Pulido et al. (2007). Three further $R_{\mathrm{C}}$ measurements from Paper I are plotted in Fig. 1.

Since measurements of $R_{\mathrm{C}}$ of the early outburst are not available in the literature, we estimated $R_{\mathrm{C}}$ from $I_{\mathrm{C}}$ measurements of Briceño et al. (2004). The $\left(R_{\mathrm{C}}-I_{\mathrm{C}}\right)$ color, as 
measured by various authors (McGehee et al. 2004; Ojha et al. 2006), seems reasonably stable during the plateau and the fading phase, showing $\left(R_{\mathrm{C}}-I_{\mathrm{C}}\right) \approx 2.0$ with a scatter of $0.2 \mathrm{mag}$. With this we calculate the expected $R_{\mathrm{C}}$ magnitudes for the period October 2003-February 2004 from the $I_{\mathrm{C}}$ measurements, assuming the same $\left(R_{\mathrm{C}}-I_{\mathrm{C}}\right)$ color for the rising phase. From Fig. 1, the optical light curve of V1647 Ori can be divided in three parts: i) from October 2003 to February 2004 - the rising period; ii) from February 2004 to August 2005 - the plateau phase and iii) from August 2005 to January 2006 - the fading period.

The rising part is very steep: from October 2003 to January 2004 the optical magnitude increased by more than 3 mag in $R_{\mathrm{C}}$. From the pre-outburst magnitude level, $R_{\mathrm{C}} \sim 23.5$, computed by McGehee et al. (see inset in Fig. 1) total rise in brightness of V1647 Ori is $>6 \mathrm{mag}$ in $R_{\mathrm{C}}$. From the light curve in Fig. 1 we find a rate of increase of $R_{\mathrm{C}}$ of $\sim 1.5 \mathrm{mag} / \mathrm{month}$. Assuming that this rate remained constant during all the rising phase, we estimate that the outburst began around the middle of August 2003, slightly earlier than October-November 2003 as found by Briceño et al. (2004).

During the plateau phase the optical brightness shows a slow decline with time $\left(\Delta R_{\mathrm{C}}=0.02 \mathrm{mag} / \mathrm{month}\right)$, on top of which $R_{\mathrm{C}}$ displays a non-periodic, flickered, oscillation on a short timescale. The light curve in Fig. 1 combines data from different works which have used different instruments and aperture sizes. This may result in a systematic scatter of the data from work to work. However, an intrinsic variation of the optical brightness on short time scales is clearly present. From our data we measure a variation of $\sim 0.5 \mathrm{mag}$ between $2004 \mathrm{Feb} .17\left(R_{\mathrm{C}}=\right.$ $16.90 \pm 0.05$, Paper I) and Feb. $18\left(R_{\mathrm{C}}=17.39 \pm 0.10\right.$, Table 1). Thus, V1647 Ori at its maximum light shows an optical brightness variation on a time scale of $24 \mathrm{~h}$. For five nights we have two consecutive acquisition images (separated by a few minutes) from which we searched for even smaller time scale variations of $R_{\mathrm{C}}$, however, no significant changes in optical brightness $\left(\Delta R_{\mathrm{C}}>0.1 \mathrm{mag}\right)$ are detectable from these measurements. The short timescale variability is of the same order as that found by other authors in the optical and near infrared (Ojha et al. 2006; Walter et al. 2004) on a timescale of a week. The total duration of the plateau phase is less than 2 years.

Our photometry confirms the rapid brightness decrease announced by Kospal et al. (2005). Four months after the claimed onset of the fading phase (August 2005, when Orion reappeared in the sky, however from the light curve it is clear that it started before), the brightness of V1647 Ori was still diminishing. From August 2005 to January $2006 R_{\mathrm{C}}$ dropped by 4 mag. On 2006 January 29 , the last $R_{\mathrm{C}}$ measurements, we estimate $R_{\mathrm{C}}=22.05 \pm 0.11$ which is still more than $1 \mathrm{mag}$ above the preoutburst level of McGehee et al. (2004). From the light curve we estimate a fading rate of $\sim 0.8 \mathrm{mag} / \mathrm{month}$ during this phase. Assuming a constant fading rate, $R_{\mathrm{C}}$ reached the pre-outburst level at the beginning of April 2006.

The light curve is not symmetric; the rising and fading phase have a different slope. From the two different rates we infer that the physical process which led to the beginning of the outburst is not simply the reverse of the process ending this outburst. The rates of brightness variation during the three phases are in agreement with those found by Acosta-Pulido et al. (2007).

\subsection{Optical spectra}

The positive slope of the optical spectrum of V1647 Ori (Fig. 2) reveals a red energy distribution of the source. Clearly visible are the $\mathrm{H} \alpha$ and $\mathrm{H} \beta$ lines both characterized by a P-Cygni profile. The He I $\lambda 5875$, Na I D1 \& D2 doublet in absorption and Fe I and Fe II lines in emission (P-Cygni) are detected in the spectra taken during the plateau phase. Due to bad pixel columns at [O I] $\lambda 5577$ and [O I] $\lambda 6300$ lines a residual of the data reduction is present in the spectra taken during the plateau phase. For this reason we cannot find evidence of any source features at these wavelengths.

During the plateau phase the optical spectrum shows only minor changes in equivalent width and line flux (see discussion below). The overall shape of the spectrum remains "constant" during this period. The spectrum taken during the fading phase (blue - 2005 December 27, red - 2006 January 29, Fig. 2) is different to all the others: superimposed on a very faint continuum with a slightly positive slope there are very strong emission lines corrisponding to $\mathrm{H} \alpha$, [O I] $\lambda \lambda 6300,6363$, [S II] $\lambda \lambda 6717$, 6731 and [Fe II] at $7172 \AA$. The sky line [O I] $\lambda 5577$ is saturated and it is not possible to find evidence of source emission at this wavelength.

From the flux-calibrated spectra, for each line detected, we computed the equivalent width $(E W)$ and the line flux $\left(F_{1}\right)$ multiplying $E W$ by the continuum level at the line center (Tables 3 and 4). A major source of uncertainties in this computation is the determination of the continuum level, which in turn depends on the accuracy of the sensitivity function and of the aperture photometry from the acquisition images. The final accuracy is of the order of $10 \%$ on $E W$ and of $15 \%$ on $F_{1}$.

The P-Cygni profile of $\mathrm{H} \alpha$ and $\mathrm{H} \beta$ is in both cases asymmetric with the emission components lacking the high velocity part (see Figs. 3, 4). This profile results from the deviation of the spherically-symmetric wind and is observed in FU Ori objects and $\mathrm{T}$ Tauri stars and can be explained by the presence of an opaque disk which occults part of the redshifted emission (see e.g. Hartmann 1998). The profiles of the two lines differ significantly (see Figs. 3 and 4): the $\mathrm{H} \beta$ has a strong and wide absorption and " "weak" narrow emission while the $\mathrm{H} \alpha$ has a large emission and a weak absorption. In both cases, the blue-shifted absorption shows at least two components: one at $\sim-450 \mathrm{~km} \mathrm{~s}^{-1}$, and the other at $\sim-150 \mathrm{~km} \mathrm{~s}^{-1}$. While the low velocity component remains almost constant over all the plateau phase, the high velocity one is variable. In particular, the latter shows in both lines a progressive decrease in extension from February 2004 to March 2005 until the whole absorption disappears in the fading phase spectrum. Furthermore, on three nights (2005 January 05, February 18 and March 15) the bluest absorption component of the $\mathrm{H} \alpha$ is "replaced" by an emission. The emission component also varies from night to night, displaying a change in equivalent width and line flux.

P Cygni signatures are also displayed by Fe lines. However, due to the low $\mathrm{S} / \mathrm{N}$ of the spectra, the absorption component is clearly detected only for the Fe II $\lambda 5169.08$ transition. The maximum absorption is at $\sim-370 \mathrm{~km} \mathrm{~s}^{-1}$ and has a terminal velocity of $\sim-600 \mathrm{~km} \mathrm{~s}^{-1}$. The other Fe emission lines show variation in equivalent width and line flux with time.

Thus, similar to what we have found for the continuum emission $\left(R_{\mathrm{C}}\right.$ magnitude), the emission lines vary on time scales of months and weeks. If continuum and line emission are produced in the same region, then the flux of a generic emission line $\left(F_{1}\right)$ is linearly proportional to continuum flux $\left(F_{\mathrm{R}}-R_{\mathrm{C}}\right.$ bandpass flux). In Fig. 5 we plotted the line flux of the strongest emission lines detected $\left(\mathrm{H} \alpha\right.$ and $\mathrm{H} \beta$ ) versus $F_{\mathrm{R}}$. Arrows indicate upper limits. Assuming a power law dependence of the line emission from $F_{\mathrm{R}}$ $\left(F_{1} \propto F_{\mathrm{R}}^{\gamma}\right)$ we searched for a correlation between line and continuum emission. The continuous line in Fig. 5 is the best fit to the 

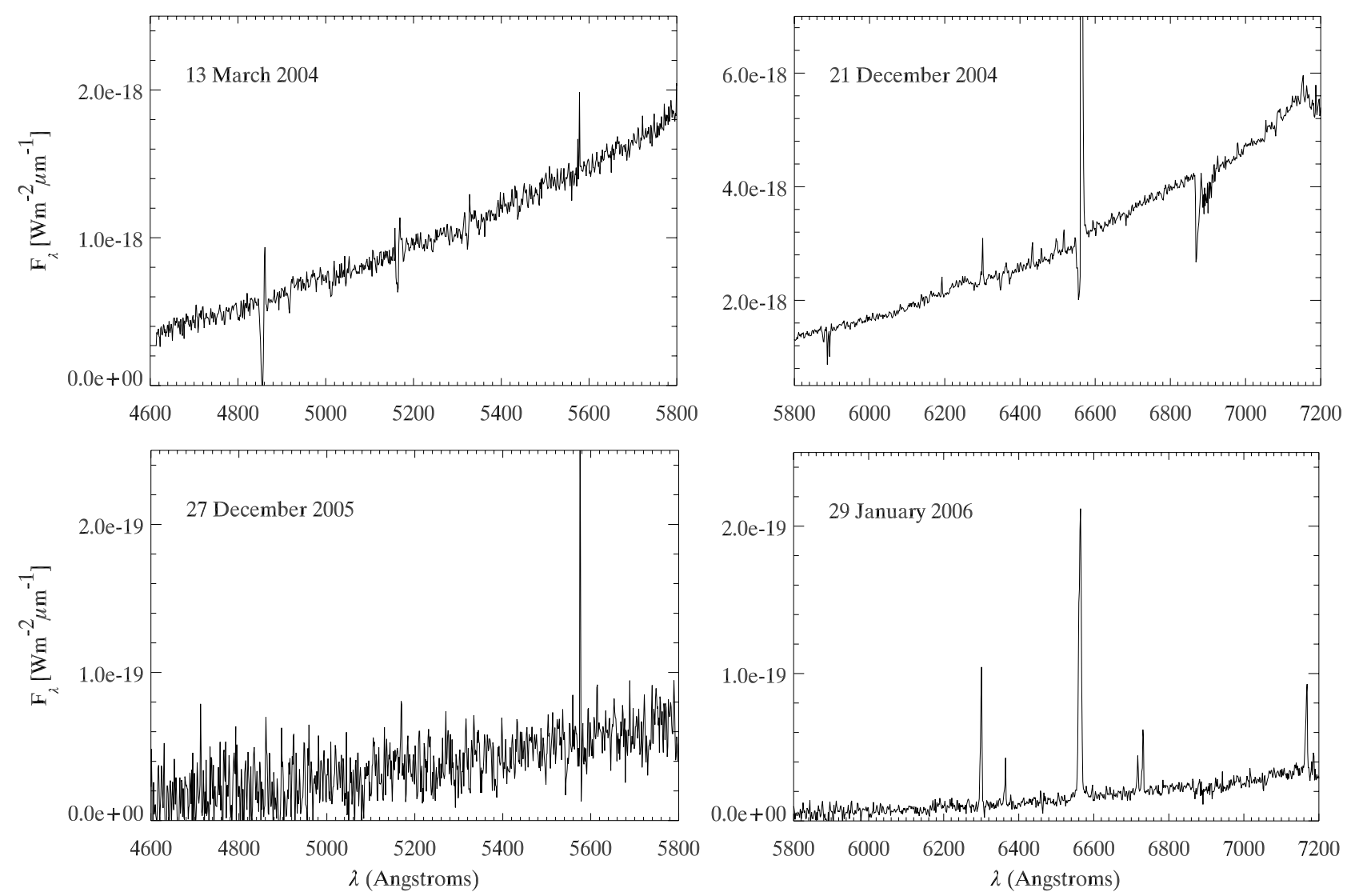

Fig. 2. Examples of optical spectra of V1647 Ori obtained during the plateau phase (top two panels), and during the fading phase (bottom two panels). Plateau spectrum: the $\mathrm{H} \alpha$ and $\mathrm{H} \beta$ are clearly visibile with a P-Cygni profile, Fe I $(5328,6191,6495 \AA)$ and Fe II (5169, 6432, 6516 $\AA$ ) lines and absorption from Na I D1 \& D2 (5889, $5895 \AA)$ and He I line (5875 $)$. A residual of the data reduction is present at [O I] (6300, $5577 \AA$ ) due to bad pixel columns, and between 6870-6910 ̊ due to a non-perfect removal of telluric absorption. Fading phase spectrum: no lines are detected in the blue part ([OI] $5577 \AA$ is a residual of the data reduction), while the red part is characterized by strong emission of $\mathrm{H} \alpha$, [O I] 6300, $6363 \AA,[S$ II] 6717, $6731 \AA$ and [Fe II] $7172 \AA$.

data. We find $\gamma_{\mathrm{H} \alpha}=0.8$ and $\gamma_{\mathrm{H} \beta}=0.7$. The $\mathrm{H} \alpha$ best fit is more robust than the $\mathrm{H} \beta$ one (the $\mathrm{H} \alpha$ emission is much stronger and it is also detected during the fading phase). All the other emission lines detected are weak and strongly affected by the low $\mathrm{S} / \mathrm{N}$ of the spectra. The power law model fit in this case is more uncertain and clear evidence of dependence of the line emission from the continuum emission cannot be found. The value of $\gamma$ found for the $\mathrm{H}$ Balmer lines is close to unity. This means that the variation in line flux is correlated to the variation of the continuum emission. This evidence might suggest that the two emissions arise from the same region of the system, or at least, that the emission mechanisms are physically linked.

Contrary to what was found by Acosta-Pulido et al. (2007), we do not find any clear trend of the Balmer emission lines with time during the plateau phase. Both the $E W$ and the line flux show a random variation with time in this period.

For the first time we detected optical forbidden lines in the spectrum of V1647 Ori taken during the fading phase (see Fig. 2, bottom right). The spectrum clearly shows strong emission from [O I] $\lambda \lambda 6300,6363,[\mathrm{~S} \mathrm{II}] \lambda \lambda 6717,6731,[\mathrm{Fe}$ II] $\lambda 7172$ an $\mathrm{H} \alpha$. These lines provide evidence for hot (a few thousand K) gas close to V1647 Ori. These are used as tracers of Herbig-Haro objects where a collimated jet from the central star collides with the ambient medium. The emission is produced by the cooling of the shocked gas. Eislöffel \& Mundt (1997) identified IRAS 05436-0007 as the driving source of HH 23. Similar and perhaps newly formed ejecta could be responsible for the
Table 2. Mid-infrared photometry of V1647 Ori from TIMMI2 and MIDI data. For comparison in Cols. 3 and 4 we report the corresponding $R_{\mathrm{C}}$ magnitude and the $R_{\mathrm{C}}-\mathrm{N} 1$ color, where $\mathrm{N} 1$ is the magnitude at $8 \mu \mathrm{m}$ converted to the ESO mid infrared photometric system (Van der Bliek et al. 1996).

\begin{tabular}{lllll}
\hline \hline DATE & $\begin{array}{l}F_{8 \mu \mathrm{m}} \\
{[\mathrm{Jy}]}\end{array}$ & $\begin{array}{l}R_{\mathrm{C}} \\
{[\mathrm{mag}]}\end{array}$ & $\begin{array}{l}R_{\mathrm{C}}-N 1 \\
{[\mathrm{mag}]}\end{array}$ & Ref. \\
\hline $2004-03-07$ & 6.4 & 16.8 & 14.4 & Muzerolle et al. (2005) \\
$2004-03-08$ & 6.5 & 16.8 & 14.4 & this work (TIMMI2) \\
$2004-03-11$ & 4.3 & 16.8 & 14.0 & Andrews et al. (2004) \\
$2004-12-31$ & 2.3 & 16.7 & 13.2 & this work (MIDI) \\
$2005-01-03$ & 2.5 & 16.7 & 13.3 & this work (MIDI) \\
$2005-02-21$ & 2.3 & 17.1 & 13.5 & this work (MIDI) \\
$2005-03-01$ & 2.5 & 17.2 & 13.7 & this work (MIDI) \\
$2006-01-10$ & 0.5 & 21.0 & 15.8 & this work (TIMMI2) \\
\hline
\end{tabular}

forbidden emission lines seen here. None of these forbidden lines were previously detected in the plateau spectrum, most likely because of the overwhelming continuum.

\subsection{Mid-Infrared spectra}

The rise in brightness of V1647 Ori during the outburst has also been seen by others authors also at longer wavelengths (see e.g. Andrews et al. 2004; Muzerolle et al. 2005; Abraham et al. 2006). Our TIMMI2 spectra (see Fig. 6) confirm the increased 
Table 3. Lines detected in the blue spectra of V1647 Ori between February 2004 and December 2005. EW is negative for emission lines and positive for absorption lines. In case of emission lines also the line flux is reported; $E W$ are expressed in $\AA$ and line flux in $10^{-18} \mathrm{~W} \mathrm{~m}^{-2}$. The accuracy on equivalent width and line flux is of the order of $10 \%$ and $15 \%$.

\begin{tabular}{|c|c|c|c|c|c|c|c|c|c|}
\hline \multirow[t]{2}{*}{ Ident. } & \multirow[t]{2}{*}{$\lambda[\AA]$} & \multicolumn{2}{|c|}{$2004-02-18$} & \multicolumn{2}{|c|}{$2004-02-23$} & \multicolumn{2}{|c|}{ 2004-03-13 } & \multicolumn{2}{|c|}{ 2004-03-18 } \\
\hline & & $E W$ & $F_{\text {line }}$ & $E W$ & $F_{\text {line }}$ & $E W$ & $F_{\text {line }}$ & $E W$ & $F_{\text {line }}$ \\
\hline $\mathrm{H} \beta(\mathrm{em})$ & 4861 & -2.91 & 1.6 & -1.94 & 1.34 & -5.38 & 3.12 & -2.22 & 1.26 \\
\hline $\mathrm{H} \beta(\mathrm{ab})$ & & 5.45 & & 5.91 & & 11.48 & & 5.68 & \\
\hline $\mathrm{Fe}$ II $(\mathrm{em})$ & 5169 & -0.58 & 0.51 & -0.17 & 0.18 & -1.68 & 1.52 & -0.37 & 0.32 \\
\hline Fe II(ab) & & 0.74 & & 1.04 & & 2.16 & & 0.98 & \\
\hline $\mathrm{Fe}_{\mathrm{I}}$ & 5328 & $>-0.7$ & $<0.78$ & -0.32 & 0.39 & -0.36 & 0.39 & $>-0.6$ & $<0.7$ \\
\hline \multirow[t]{2}{*}{ Ident. } & $\lambda[\AA]$ & \multicolumn{2}{|c|}{ 2004-03-27 } & \multicolumn{2}{|c|}{ 2004-12-08 } & \multicolumn{2}{|c|}{ 2004-12-21 } & \multicolumn{2}{|c|}{ 2005-01-05 } \\
\hline & & $E W$ & $F_{\text {line }}$ & $E W$ & $F_{\text {line }}$ & $E W$ & $F_{\text {line }}$ & $E W$ & $F_{\text {line }}$ \\
\hline $\mathrm{H} \beta(\mathrm{em})$ & 4861 & -1.22 & 0.75 & -3.33 & 2.08 & -2.75 & 2.02 & -2.00 & 1.27 \\
\hline $\mathrm{H} \beta(\mathrm{ab})$ & & 5.53 & & 5.23 & & 6.05 & & 2.15 & \\
\hline Fe II (em) & 5169 & $>-1.2$ & $<1.2$ & -0.70 & 0.62 & -0.49 & 0.53 & $>-1.3$ & $<1.2$ \\
\hline $\mathrm{Fe}$ II (ab) & & 1.34 & & $<1.9$ & & $<1.2$ & & 0.59 & \\
\hline $\mathrm{Fe} \mathrm{I}$ & 5328 & -0.30 & 0.34 & $>-1.1$ & $<1.2$ & -0.29 & 0.38 & -0.54 & 0.62 \\
\hline \multirow[t]{2}{*}{ Ident. } & $\lambda[\AA]$ & \multicolumn{2}{|c|}{ 2005-02-18 } & \multicolumn{2}{|c|}{ 2005-02-29 } & \multicolumn{2}{|c|}{$2005-03-15$} & \multicolumn{2}{|c|}{$2005-12-27$} \\
\hline & & $E W$ & $F_{\text {line }}$ & $E W$ & $F_{\text {line }}$ & $E W$ & $F_{\text {line }}$ & $E W$ & $F_{\text {line }}$ \\
\hline $\mathrm{H} \beta(\mathrm{em})$ & 4861 & -4.22 & 1.53 & $>-1.5$ & $<0.94$ & -1.61 & 0.70 & $>-11.1$ & $<0.2$ \\
\hline $\mathrm{H} \beta(\mathrm{ab})$ & & $<6.2$ & & 2.55 & & 2.83 & & $<22.1$ & \\
\hline Fe II (em) & 5169 & $>-2.7$ & $<1.5$ & $>-1.2$ & $<1.1$ & $>-1.1$ & $<0.8$ & $>-4.9$ & $<0.2$ \\
\hline $\mathrm{Fe}$ II (a & & $<4.1$ & & $<1.9$ & & $<1.7$ & & $<7.3$ & \\
\hline $\mathrm{Fe} I$ & 5328 & $>-2.2$ & $<1.4$ & $>0.8$ & $<0.8$ & $>-1.3$ & $<1.0$ & $>-4.5$ & $<0.2$ \\
\hline
\end{tabular}

Table 4. Continuation of Table 3 for red spectra between December 2004 and January 2006.

\begin{tabular}{|c|c|c|c|c|c|c|c|c|c|}
\hline \multirow[t]{2}{*}{ Ident. } & \multirow[t]{2}{*}{$\lambda[\AA]$} & \multicolumn{2}{|c|}{ 2004-12-08 } & \multicolumn{2}{|c|}{$2004-12-21$} & \multicolumn{2}{|c|}{ 2005-01-05 } & \multicolumn{2}{|c|}{$2005-02-18$} \\
\hline & & $E W$ & $F_{\text {line }}$ & $E W$ & $F_{\text {line }}$ & $E W$ & $F_{\text {line }}$ & $E W$ & $F_{\text {line }}$ \\
\hline$\overline{\mathrm{He}} \mathrm{I}$ & 5875 & 1.14 & & 0.71 & & 0.32 & & $<1.6$ & \\
\hline $\mathrm{Na}$ I (D1) & 5889 & 1.44 & & 0.97 & & 2.26 & & 2.82 & \\
\hline $\mathrm{Na}$ I (D2) & 5895 & 1.01 & & 0.70 & & 1.17 & & 2.88 & \\
\hline $\mathrm{Fe}$ I (169) & 6191 & -0.31 & 0.54 & -0.35 & -0.74 & -0.34 & 0.63 & $>0.08$ & $<0.8$ \\
\hline [O I] & 6300 & - & - & - & - & - & - & - & - \\
\hline [O I] & 6363 & - & - & - & - & - & - & - & - \\
\hline $\mathrm{Fe}$ II & 6432 & -0.74 & 1.58 & -0.44 & 1.17 & -0.45 & 1.03 & -0.48 & 0.58 \\
\hline $\mathrm{Fe} \mathrm{I}$ & 6495 & -1.08 & 2.46 & -0.55 & 1.54 & -0.73 & 1.76 & -0.83 & 1.08 \\
\hline $\mathrm{Fe}$ II & 6516 & -0.55 & 1.27 & -0.43 & 1.24 & -0.53 & 1.30 & -0.69 & 0.93 \\
\hline $\mathrm{H} \alpha(\mathrm{em})$ & 6562 & -26.61 & 66.34 & -28.74 & 88.66 & -30.27 & 80.44 & -38.81 & 56.60 \\
\hline $\mathrm{H} \alpha(\mathrm{ab})$ & & 3.30 & & 2.55 & & 0.74 & & $<0.53$ & \\
\hline [S II] & 6717 & - & - & - & - & - & - & - & - \\
\hline [S II] & 6731 & - & - & - & - & - & - & - & - \\
\hline$[\mathrm{Fe} \mathrm{II}]$ & 7172 & - & - & - & - & - & - & - & - \\
\hline \multirow[t]{2}{*}{ Ident. } & $\lambda[\AA]$ & \multicolumn{2}{|c|}{$2005-02-29$} & \multicolumn{2}{|c|}{ 2005-03-15 } & \multicolumn{2}{|c|}{ 2006-01-29 } & & \\
\hline & & $E W$ & $F_{\text {line }}$ & $E W$ & $F_{\text {line }}$ & $E W$ & $F_{\text {line }}$ & & \\
\hline $\mathrm{He} \mathrm{I}$ & 5875 & $<0.8$ & & $<1.1$ & & $<10.9$ & & & \\
\hline Na I (D1) & 5889 & 1.87 & & 1.16 & & $<9.1$ & & & \\
\hline $\mathrm{Na}$ I (D2) & 5895 & 0.87 & & 1.51 & & $<7.3$ & & & \\
\hline $\mathrm{Fe}$ I (169) & 6191 & -0.24 & 0.44 & -0.30 & 0.38 & $>-3.3$ & $<0.03$ & & \\
\hline [O I] & 6300 & - & - & - & - & -40.98 & 0.42 & & \\
\hline [O I] & 6363 & - & - & - & - & -6.92 & 0.08 & & \\
\hline $\mathrm{Fe}$ II & 6432 & -0.42 & 0.94 & -0.54 & 0.85 & $>-2.7$ & $<0.03$ & & \\
\hline $\mathrm{Fe} \mathrm{I}$ & 6495 & -0.52 & 1.23 & -0.92 & 1.59 & $>-2.7$ & $<0.03$ & & \\
\hline $\mathrm{Fe}$ II & 6516 & -0.35 & 0.85 & -0.52 & 0.92 & $>-2.7$ & $<0.03$ & & \\
\hline $\mathrm{H} \alpha(\mathrm{em})$ & 6562 & -17.25 & 44.65 & -22.07 & 39.94 & -108.30 & 1.66 & & \\
\hline $\mathrm{H} \alpha(\mathrm{ab})$ & & 1.78 & & 1.10 & & $<3.9$ & & & \\
\hline [S II] & 6717 & - & - & - & - & -4.52 & 0.09 & & \\
\hline [S II] & 6731 & - & - & - & - & -7.24 & 0.15 & & \\
\hline [Fe II $]$ & 7172 & - & - & - & - & -9.48 & 0.32 & & \\
\hline
\end{tabular}

mid-infrared flux: from the pre-outburst level of $0.53 \mathrm{Jy}$ at $12 \mu \mathrm{m}$ (IRAS, Point Source Catalog) up to 7.6 Jy on 2004 March 08. The $8-14 \mu \mathrm{m}$ spectrum is essentially featureless and flat all along the spectral range. This result is not consistent with the mid-infrared spectrum in Andrews et al. (2004) taken with
UKIRT/Michelle only three days after our TIMMI2 spectrum. Their measurement reveals a strong red energy distribution with the flux going from $4.0 \mathrm{Jy}$ at $8 \mu \mathrm{m}$ up to $12.0 \mathrm{Jy}$ at $12 \mu \mathrm{m}$. Our estimate of the mid-infrared flux is however consistent with the SPITZER/IRAC observations of Muzerolle et al. (2005) taken 
D. Fedele et al.: Temporal evolution of the outburst of V1647 Ori. II.
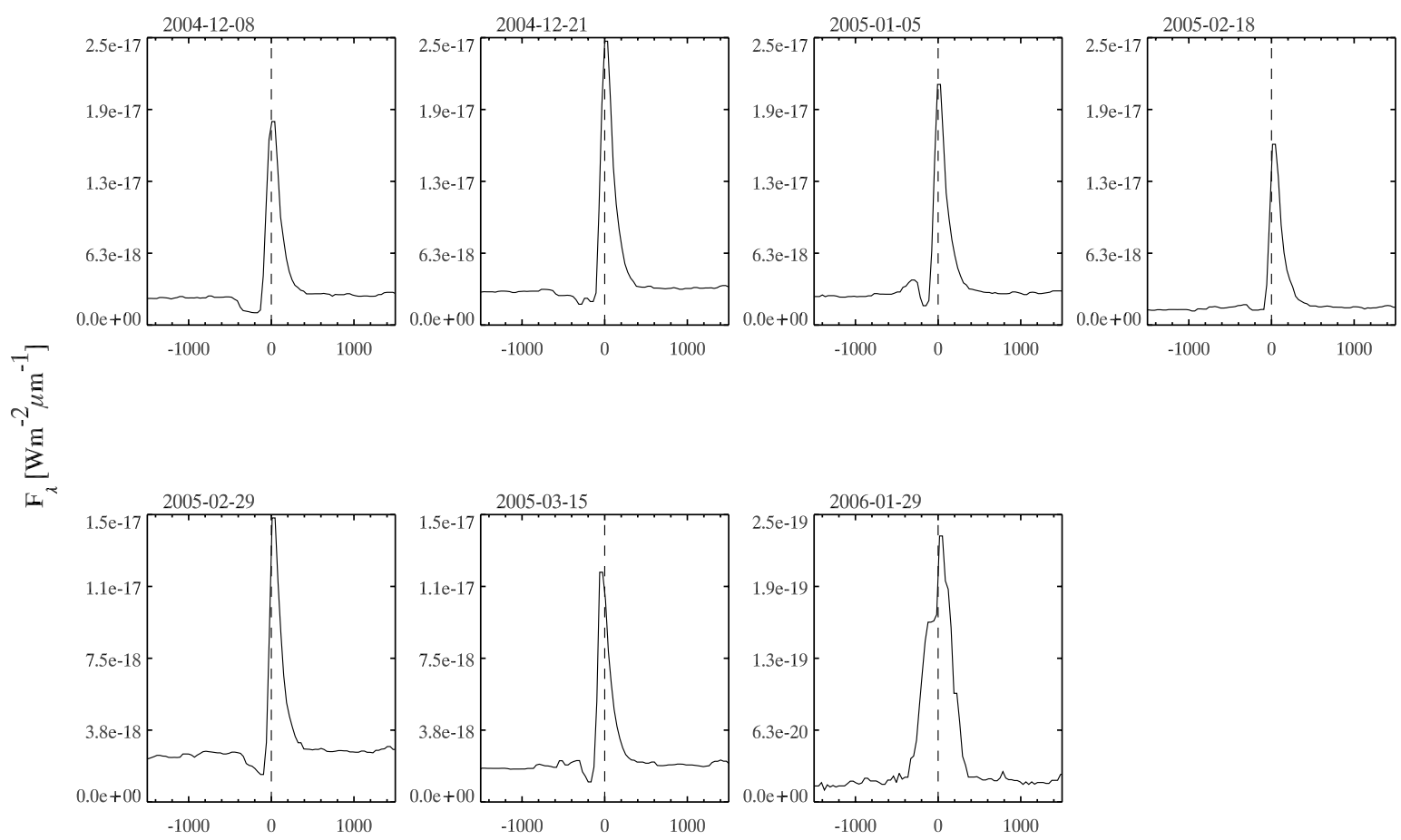

$\mathrm{v}\left[\mathrm{km} \mathrm{s}^{-1}\right]$

Fig. 3. Time evolution of the $\mathrm{H} \alpha$ emission in the period December 2004-January 2006. In all the "plateau spectra" the line has an asymmetric P-Cygni profile. In the "fading phase spectrum", the line is pure in emission with no trace of absorption.
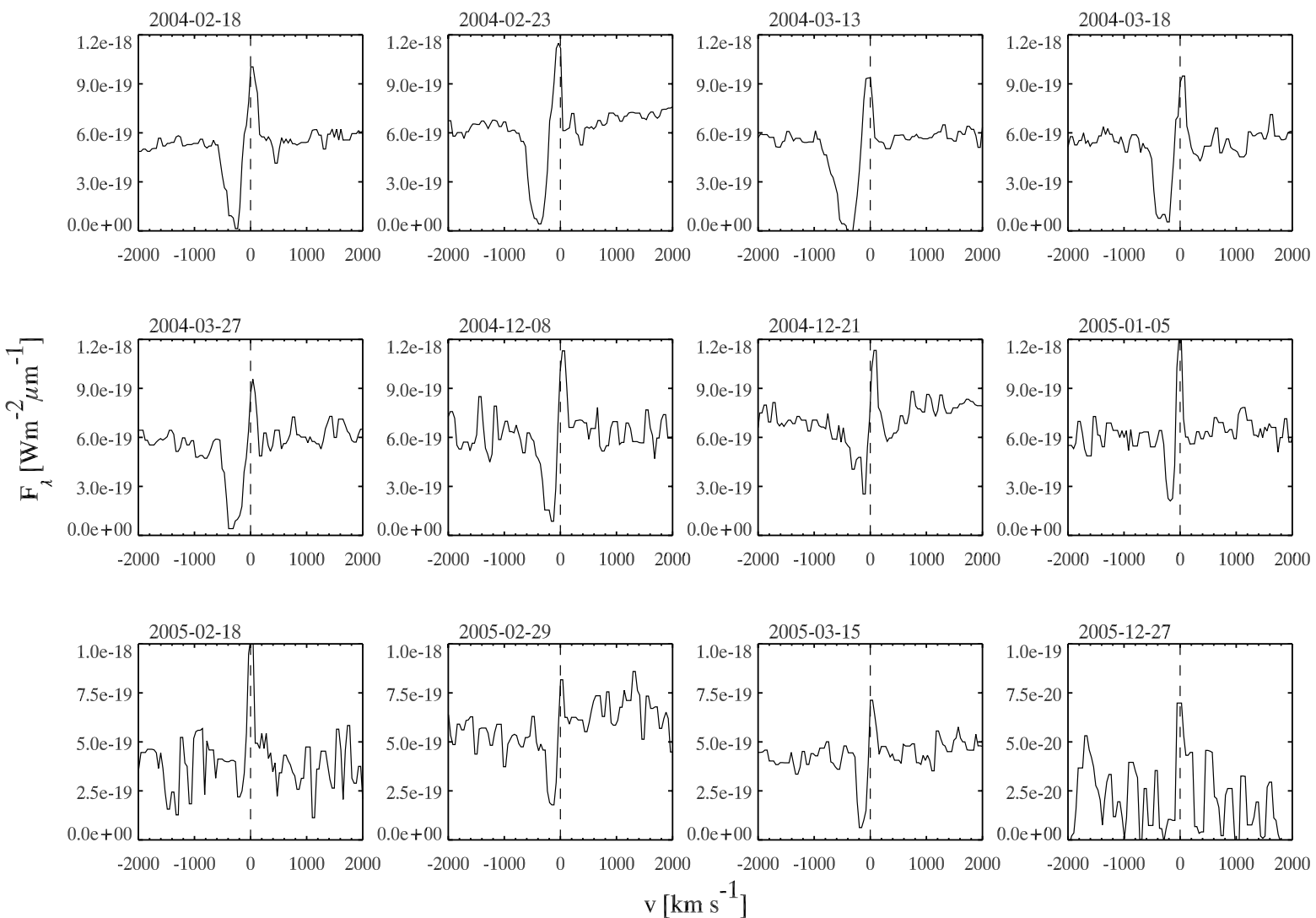

Fig. 4. Time evolution of the $\mathrm{H} \beta$ emission in the period February 2004-December 2005. As for the $\mathrm{H} \alpha$, during the plateau phase the line has a $\mathrm{P}$-Cygni profile. No $\mathrm{H} \beta$ emission is detected in the low $\mathrm{S} / \mathrm{N}$ "fading phase spectrum". 

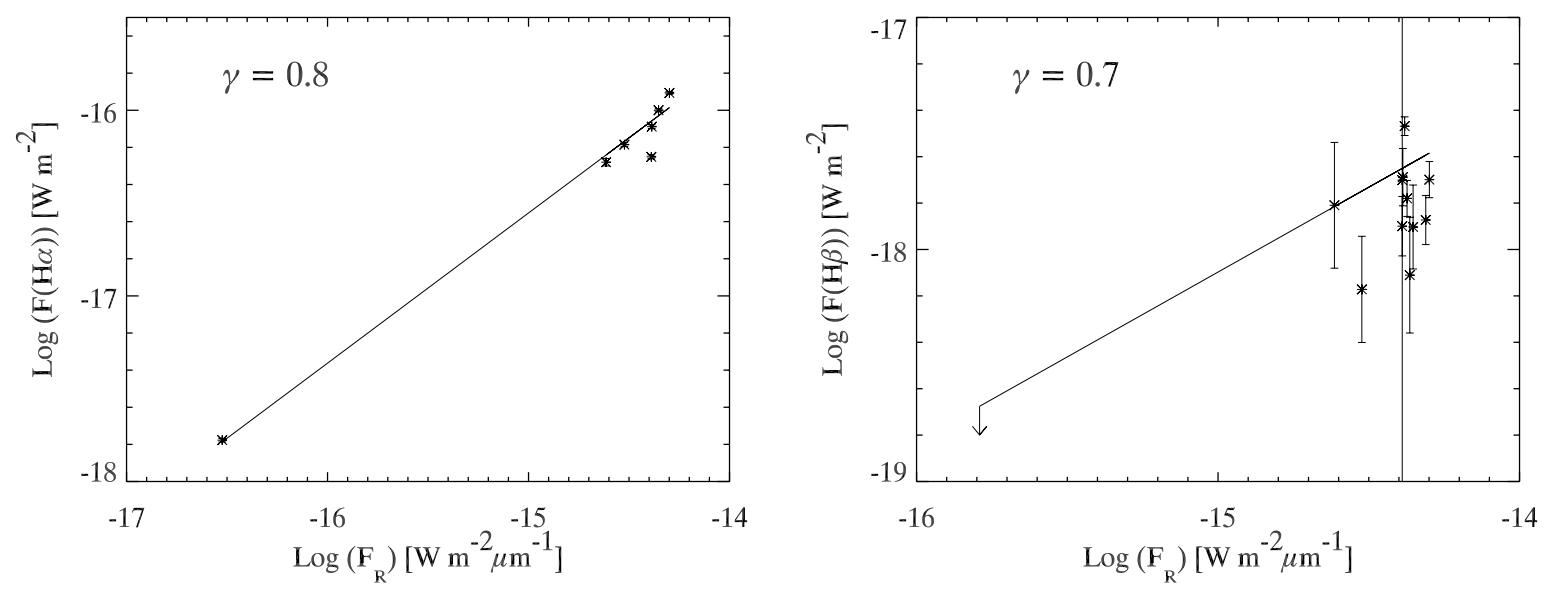

Fig. 5. Line flux of $\mathrm{H} \alpha$ and $\mathrm{H} \beta$ versus continuum flux. The best power law fit is over-plotted.

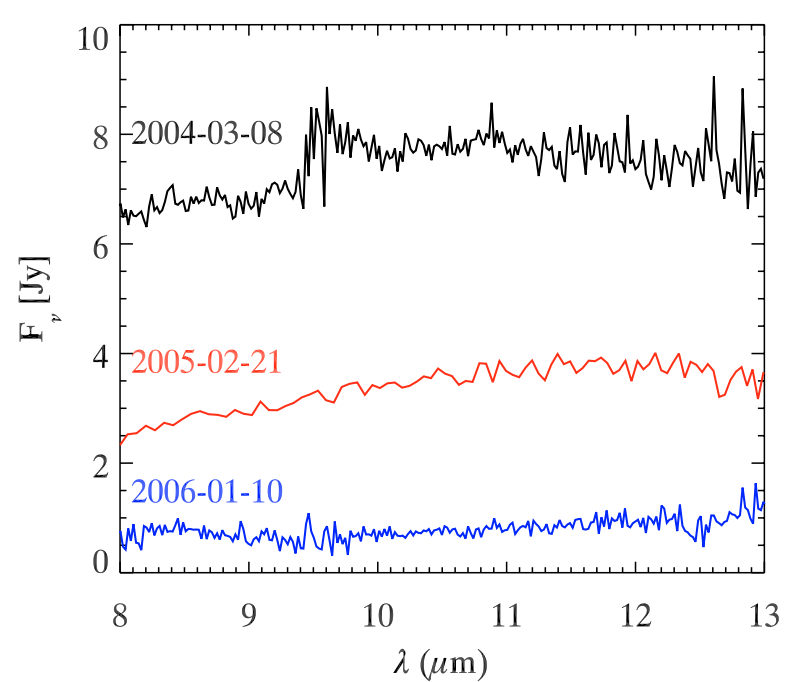

Fig. 6. Time evolution of the mid-Infrared spectrum of V1647 Ori taken with TIMMI2 (black and blue lines) and MIDI (red line). The midinfrared spectrum measured by MIDI remain constant, within the uncertainties (10\%), between December 2004 and March 2005. Here, only the MIDI spectrum taken on 2005 February 21 is shown.

on 2004 March 07. They measure a flux of $6.43 \mathrm{Jy}$ at $8 \mu \mathrm{m}$, very close to our estimate. A residual of the data reduction corresponding to the strong atmospheric absorption bands centered at $9.6\left(\mathrm{O}_{3}\right)$ and $12.55 \mu \mathrm{m}\left(\mathrm{CO}_{2}\right)$ do not allow us to better analyze these two regions.

Ten months later, in December 2004, the mid-infrared flux of V1647 Ori revealed by the MIDI observations dropped by a few Jy. The spectrum is again flat and featureless. The MIDI spectra analyzed were all taken during the optical plateau phase (Fig. 6). They all reveal a flat and featureless spectrum. Within the accuracy of these spectra $(10 \%)$, the flux level remains constant between December 2004 and March 2005. Thus, also in mid-IR the system experienced a plateau phase.

The rapid optical brightness fading is also experienced by the system in the mid-infrared: on 2006 January 11, the flux level of our TIMMI 2 spectrum at $12 \mu \mathrm{m}$ is $0.9 \mathrm{Jy}$, still considerably higher than the pre-outburst level. Also in this case the spectrum is flat and featureless.

The optical and mid-IR light curve of V1647 Ori are different. In the optical the brightness increases during the rising phase, remaining below the value of the plateau phase and

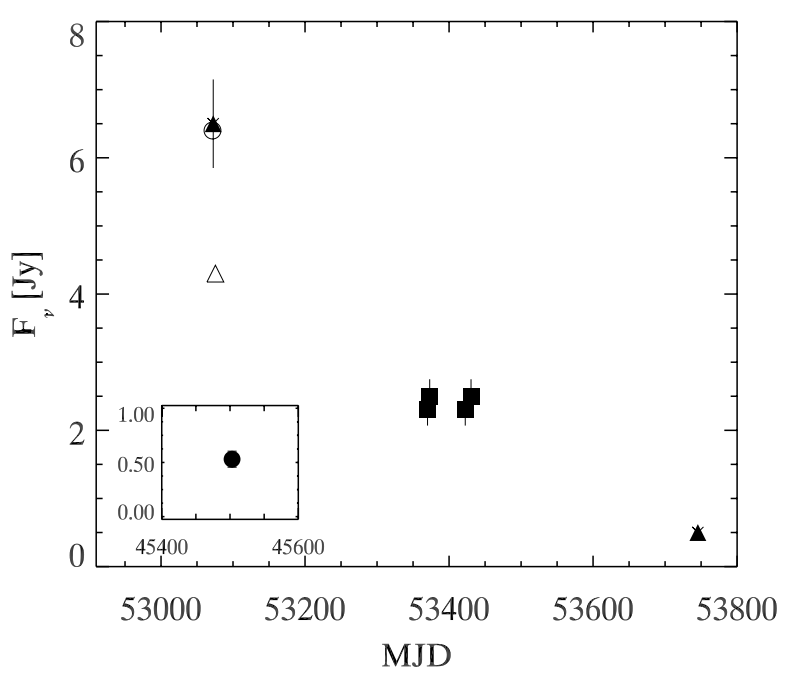

Fig. 7. $8 \mu \mathrm{m}$ light curve of V1647 Ori. Filled triangles - TIMMI2 data; filled squares - MIDI data; open circle - SPITZER/IRAC data from Muzerolle et al. (2005); open triangle - UKIRT/MICHELLE data from Andrews et al. (2004). The inset shows the pre-outburst IRAS measurements at $12 \mu \mathrm{m}$.

finally decreases. On the other hand, the mid-IR brightness is higher during the "mid-IR rising phase" than during the "mid-IR plateau phase" (Fig. 7).

\section{Discussion}

Pre-main-sequence stars are known to be intrinsically variable objects. The variability mechanisms might be different: solarlike coronal activity, spots on the stellar surface, stellar pulsation, partial obscuration and subsequent clearing of the line of sight. These processes are however unable to generate the $\sim 44 L_{\odot}$ luminosity increment produced by V1647 Ori and to produce the $\gtrsim 6$ optical magnitude burst lasting more than 2 years. To release such an amount of energy the existence of a secondary luminosity source is necessary. Similar brightening events from FU Orionis stars are explained by a sudden increase of the mass accretion rate from a circumstellar disk onto the central star. The increased accretion rate produces an accretion luminosity $\left(L_{\text {acc }} \propto \dot{M}\right)$ which may overwhelm the stellar brightness. Such a process can explain both the dramatic brightening (from X-ray to the infrared) as well as the strong $\mathrm{H} \alpha$ emission observed in the 
recent outburst of V1647 Ori. Kastner et al. (2006) confirmed that the X-ray evolution of V1647 Ori in outburst reflects the near-infrared evolution and is consistent with the hypothesis of an increased mass accretion rate.

As a consequence of the enhanced accretion rate a strong wind develops from the disk's surface. The blue-shifted absorption components of $\mathrm{H} \alpha$ and $\mathrm{H} \beta$ in the spectrum of V1647 Ori are signatures of this wind. The disappearance of the absorption component in $\mathrm{H} \alpha$ during the fading phase is a confirmation that the strong wind ceased and that the system has been going back to a phase of slow accretion. In this system the origin of the $\mathrm{H}$ Balmer emission lines is controversial since both the wind and the mass infall might contribute to the line formation. The magnetospheric accretion predicts a further contribution to the line emissivity produced in the optically thin free-falling accretion columns.

The Fe II lines at 6432.68 and $6516.81 \AA$ detected in the spectra of V1647 Ori both originate from multiplet 40. They are commonly also seen in emission in the spectra of strongly accreting young stars such as V380 Ori (Rossi 1999; Shevchenko 1999), Z CMa (Hessman et al. 1991; Garcia et al. 1999; van den Ancker et al. 2004), PV Cep and MWC 1080 (Hernandez et al. 2004) and are amongst the strongest emission lines in such environments. These Fe II lines appear to be absent from the spectrum of young stars showing lower accretion rates. The presence of Fe I 6495.81 emission and the absence of strong Fe II lines from higher multiplets suggests that the ironline forming region in V1647 Ori may be somewhat cooler than the typical electron temperatures of 10000-20000 K inferred in for example Z CMa (van den Ancker et al. 2004).

\subsection{The circumstellar envelope and the puzzling mid infrared spectrum}

The accretion disk alone is not able to produce the long wavelength $(\lambda \gtrsim 10 \mu \mathrm{m})$ emission observed, unless it flares strongly over a large range of distance scales (see e.g. Hartmann 1998). The sub-millimeter continuum flux during the outburst remains at its pre-outburst level and there are no signatures of flux changes in these wavelength regimes (Andrews et al. 2004). These findings are consistent with the presence of a dusty circumstellar envelope, probably a remnant of the infalling envelope. Intriguingly, Kenyon \& Hartmann (1991) suggested the presence of the infalling envelope to explain the enhanced midinfrared flux from FU Orionis objects in the eruptive phase.

Muzerolle et al. (2005, hereafter M04) attempt to reproduce the spectral energy distribution (SED) of V1647 Ori by means of a standard viscous accretion disk and of an optically thin envelope. Their model predicts a $10 \mu \mathrm{m}$ emission feature that is produced by silicate dust grains. However, our multiepoch mid-infrared spectroscopy reveals a flat and featureless spectrum during the whole outburst duration (see Fig. 6). This is highly unusual. In FU Orionis objects the silicate feature is seen sometimes in emission (V1057 Cyg, FU Ori, BBW 76, V1515 Cyg, Green et al. 2006) and sometimes in absorption (V346 Nor, Z CMA Green et al. 2006; Acke \& van den Ancker 2004). These differences are probably caused by differences in the optical thickness of the system (disk + envelope) at $10 \mu \mathrm{m}$.

The model proposed by Abraham et al. (2006) adopts a simple viscous accretion disk model (without envelope). Their model predicts a flat and featureless mid infrared spectrum. However, the emission at longer wavelength $(\gtrsim 10 \mu \mathrm{m})$ requires an highly flared disk. In such a model the outer part of the disk is directly illuminated during the outburst producing a flux enhancement also in the (sub-)millimeter. This is not observed (Andrews et al. 2004). The stability of the (sub-)millimeter emission favors the presence of a dusty circumstellar envelope.

The excitation plot for the $\mathrm{CO}$ fundamental ro-vibrational lines (Rettig et al. 2005) and the detection of $\Delta v=2 \mathrm{CO}$ band heads at $\sim 2.3 \mu \mathrm{m}$ (Vacca et al. 2004) indicate the presence of hot $(\sim 2500 \mathrm{~K})$ and dense gas. Since the dust sublimes at $\sim 1500 \mathrm{~K}$, such emission likely arises in regions free of dust. Our suggestion is that even in the mid infrared the bulk of the emission is produced by the gas in a dust-free region of the disk. Nevertheless we cannot exclude a contribution from the dust (e.g. arising at larger radii in the disk). The emission at longer wavelengths is dominated by the dust in the envelope.

Figure 7 shows the temporal evolution of the mid infrared flux during the outburst. The flux decay at these wavelengths is faster than the decay in the optical (compare with Fig. 1). The 8-14 $\mu \mathrm{m}$ flux drastically decreases from March to December 2004 while the optical continuum remains constant over the same period. This produces radical changes in the SED of V1647 Ori during the outburst. As can be seen from the $R_{\mathrm{C}}-\mathrm{N} 1$ color (Table 2) the system is redder during the early outburst. A likely explanation is that in the earlier phases of the outburst the disk was hotter and a larger region of the disk contributed to the emission seen at $10 \mu \mathrm{m}$. However, a detailed modeling of the observations is necessary to explain which parameters (such as temperature, opacity, surface area) are responsible for the observed changes.

\section{Conclusion}

Outbursts in pre-main-sequence stars historically have been classified in two main groups based upon their similarity to the prototypes FU Orionis and EX Lupi (Herbig 1977) depending on outburst duration, maximum magnitude variation and spectral features at maximum light. Table 5 lists the main characteristics of the two groups and that of V1647 Ori.

Since the onset of the outburst of V1647 Ori it has been debated whether this system is either as FUor or an EXor object. V1647 Ori resembles some aspects of an EXor (outburst duration, recurrence of the outburst), and some aspects of an FUor (magnitude rise, SED). However the recurrence timescale of the outburst has an intermediate value between the two classes. Its emission line spectrum is clearly distinct from either the absorption line spectrum of an FUor or the T Tauri-like spectrum of an EXor (where the $\mathrm{H}$ lines show as inverse $\mathrm{P}-\mathrm{Cygni}$ profile).

V1647 Orionis is not the only outburst PMS suspected of having a nature intermediate between the two main classes. OO Ser recently experienced an outburst that lasted $\sim 5-10$ years (Kospal et al. 2006), too fast for an FUor and too slow for an EXor. The SED of OO Ser is typical of an FU Orionis object, and has roughly the same shape in quiescent and outburst phase.

A common denominator in all young eruptive stars detected so far seems to be the presence of circumstellar material as well as that of a reflection nebula. These structures are likely remnants of the infalling envelope. The infalling envelope is a potential reservoir of mass for the disk which experiences recursive outbursts. Assuming that $L_{\mathrm{bol}}$ during the outburst is dominated by the accretion luminosity, M04 estimate a mass accretion rate of $\sim 10^{-5} M_{\odot} \mathrm{yr}^{-1}$. Considering the $2-3$ year duration of the outburst and the 37 year recurrence timescale, a constant envelope infall rate of $\sim 7 \times 10^{-7} M_{\odot} \mathrm{yr}^{-1}$ is necessary to replenish the disk after each outburst. The disk accretion rate during the quiescent phase is estimated to be $\sim 6 \times 10^{-7} M_{\odot} \mathrm{yr}^{-1}$ (see e.g. M04). 
Table 5. Typical values of outburst from pre-main-sequence stars. The outburst recurrence of FU Orionis objects has been estimated as the time needed to replenish the disk mass after an outburst with a constant infall rate.

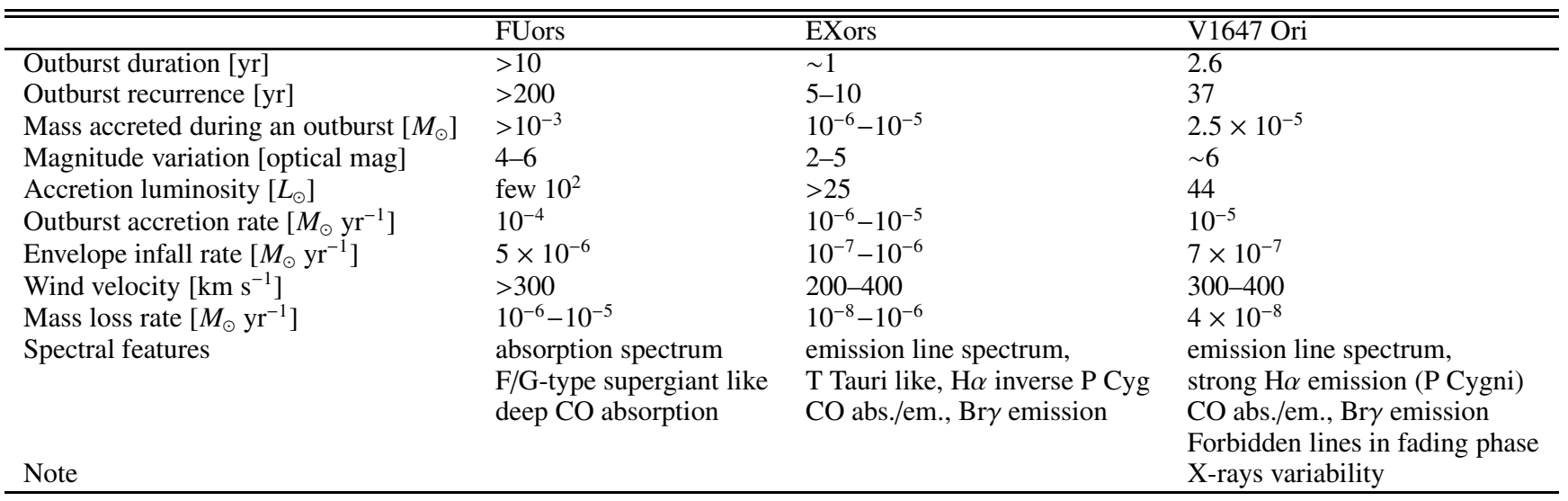

Submillimeter maps reveal that FU Orionis stars have accretion disks that are larger and more massive than those of $\mathrm{T}$ Tauri stars (Sandell \& Weintraub 2001) and are comparable in mass to those seen around Class I sources (i.e. young stellar objects with flat or rising infrared SED). The circumstellar material around V1647 Ori accounts for $0.04 \pm 0.01 M_{\odot}$ (Tsukagoshi et al. 2005) which is slightly larger than the disk mass of a T Tauri star $\left(\sim 0.01 M_{\odot}\right)$. All these findings suggest that outbursts occur in Class I sources, where the star is still embedded in the infalling envelope. The outburst duration and mass accretion rate during outburst seem to correlate with the infall rate (see Table 5): objects with higher infall rates have longer outbursts and reach higher accretion rates while objects with smaller infall rates experience short-lived outbursts. The occurrence of short outbursts might suggest that the envelope is becoming thinner, i.e. that the system is in a transition phase of an embedded Class I source to an optically visible star surrounded by a protoplanetary disk (Class II).

The Orion Nebula Cluster (ONC) is subject to extensive observational campaigns. So far, roughly 1600 stars have been confirmed to be members of the ONC and 55\% of these (at least) posses a circumstellar disk (Hillenbrand et al. 1998). Assuming that Class I sources account for $20 \%$ of stars with disks (lower limit, equal to the Class I fraction found in the original paper of Lada \& Wilking 1984), we expect to have more than 200 Class I sources in the ONC only. If all of these 200 sources experience FU Orionis-like activity, we would expect to see 10-40 (depending on outburst duration and recurrence of FUors, V1647 Ori and EXors) of these stars in outburst at any time in the ONC. The total number of young eruptive stars discovered so far in the whole Orion star forming region is only seven: three FUors (FU Orionis itself, V883 Ori and Reipurth 50), three EXors (NY Ori, V1118 Ori, V1143 Ori) and V1647 Ori. Not all of these are in outburst at the same time. It appears that there is a deficit of observed outbursts in Orion. We conclude that not all Class I sources undergo FU Orionis-like events for their entire lifetime.

A possible solution to this problem is that we are overestimating the number of "true" Class I sources in Orion. The spectral energy distribution of an isolated $\mathrm{T}$ Tauri source seen with a disk close to edge-on may mimic a typical Class I SED. An alternative solution is that outbursts occur only in a specific stage of early evolution, namely, in the transition phase of an embedded Class I source to an optically visible T Tauri or Herbig AeBe star.
We also caution that this result is based only on a small number of Class I sources in Orion, namely, those not embedded in high density regions. Recent Spitzer observations show that there is a large number of such sources which lie in regions of extremely high extinction (Megeath et al. 2006). An outburst in one of these objects could easily have been missed in the existing surveys of Orion. More regular infrared surveys of star forming regions are needed to investigate the number of FUor-like outbursts in these embedded sources.

\subsection{Outburst mechanism}

Instability mechanisms of different flavors have been proposed to explain the FU Orionis phenomenon. The gravitational forces of a companion star may perturb the disk, enhancing accretion (see e.g. Bonnell \& Bastien 1992). Gravitational instability has been also proposed but, for this instability to occur the disk has to be cold and massive. The most accepted mechanism to trigger such outbursts is via thermal instability in the inner disk (see e.g. Lin \& Papaloizou 1985; Clarke et al. 1990; Kawazoe \& Mineshige 1993; Bell et al. 1995). A key point of this model is that of a high accretion rate in the outer disk, of the order of few $\times 10^{-6} M_{\odot} \mathrm{yr}^{-1}$. According to this model, outbursts will occur as long as mass is deposited in the outer disk at such a high rate. This implies that the outbursts will become shorter in time and smaller in amplitude as the infall into the disk ceases. An alternative explanation for eruptive events has been suggested by Gammie (1996). He suggested that "dead zones" of decreased accretion may develop in the case of magnetic viscosity. Material from the outer part of the disk may accumulate at the edge of such dead zones until high-accretion-rate episodes occur.

One way to explain the different outburst properties of FUors, EXors and V1647 Ori is by introducing a different outburst mechanism for each of these classes of objects. However, the data collected so far in all young eruptive stars raise the possibility of the existence of a unique class of outburst objects. In this scenario, the different types of outburst are produced by a continuum variation of one or more of the parameters involved in the instability, rather than a variation of physical mechanisms. For instance, the presence of a remnant of the infalling envelope and the estimated infall rate (see Table 5) might favor thermal instability as the only model to explain the three groups. If this is the case, the different outburst durations between an FU Orionis, V1647 Ori and an EX Lupi might be explained by the difference 
in the infall rate. The thermal instability model indeed predicts shorter and smaller amplitude outbursts as infall ceases below $10^{-6} M_{\odot} \mathrm{yr}^{-1}$, to approach typical T Tauri disk accretion rate of $\sim 10^{-7} M_{\odot} \mathrm{yr}^{-1}$.

The same model is also able to explain the diversity in spectral features of the three classes of objects. The mass accretion rate reached during the outburst is (also) dependent on the envelope infall rate. When very high accretion rates and disk temperatures are reached, as in the case of FUors, the disk's interior may become hotter than the disk surface and the emitted spectrum will show absorption lines. If the accretion rate is not high enough to invert the temperature gradient in the disk's interior, the final spectrum will be dominated by emission features which are produced in the disk's wind and/or in the magnetospheric accretion columns. In this case we will see EXors or V1647 Ori-like spectra.

Acknowledgements. The authors thank the ESO Paranal and La Silla staff for performing the service mode observations.

\section{References}

Abraham, P., Kospal, A., Csizmadia, A., et al. 2004, A\&A, 419, L39 Abraham, P., Mosoni, L., Henning, T., et al. 2006, A\&A, 449, L13 Acke, B., \& van den Ancker, M. E. 2004, A\&A, 426, 151

Acosta-Pulido, J. A., Kun, M., Ábrahám, P., et al. 2007, AJ, 133, 2020

Andrews, S. M., Rothberg, B., \& Simon, T. 2004, ApJ, 610, L45

Appenzeller, I., et al. 1998, The Messenger, 94, 1

Aspin, C., Barbieri, C., Boschi, F., et al. 2006, AJ, 132, 1298

Bell, K. R., Lin, D. N. C., Hartmann, L. W., \& Kenyon, S. J. 1995, ApJ, 444, 376

Bonnel, I., \& Bastien, P. 1992, ApJ, 401, 654

Briceño, C., Vivas, A. K., Hernandez, J., et al. 2004, ApJ, 606, L123

Clarke, C., Lin, B. D. C., \& Pringle, J. E. 1990, MNRAS, 242, 439

Clarke, C., Lodato, G., Melnikov, S. Y., \& Ibrahimov, M. A. 2005 MNRAS, 361, 942

Cohen, M., Walker, R. G., Carter, B., et al. 1999, AJ, 117, 1864

Eislöffel, J., \& Mundt, R. 1997, AJ, 114, 280

Fedele, D., van den Ancker, M. E., Petr-Gotzens, M. G., Ageorges, N., \& Rafanelli, P. 2007, 472, 199

Gammie, C. F. 1996, ApJ, 457, 355
Garcia, P. J. V., Thiébaut, E., Bacon, R., et al. 1999, A\&A, 346, 892 Green, J. D., Hartmann, L., Calvet, N., et al. 2006, ApJ, 648, 1099 Grosso, N., Kastner, J. H., Ozawa, H., et al. 2005, A\&A, 438, 159 Hartmann, L., \& Kenyon, S. J. 1996, ARA\&A, 34, 207

Hartmann, L. 1998, Accretion processes in star formation

Herbig, G. H. 1966, Vistas Astron., 8, 10

Herbig, G. H. 1977, ApJ, 217, 693

Hernandez, J., Calvet, N., Briceño, C., et al. 2004, AJ, 127, 1682

Hessman, F. V., Eisloeffel, J., Mundt, R., et al. 1991, ApJ, 370, 384

Hillenbrand, L. A., Strom, S. E., Calvet, N., et al. 1998, AJ, 116, 1816

Kastner, J. H., Richmond, M., Grosso, N., et al. 2006, ApJ, 648, L43

Kawazoe, E., \& Mineshige, S. 1993 PASP, 45, 715

Käufl, H.-U., Sterzik, M. F., Siebenmorgen, R., et al. 2003, SPIE, 4841, 117

Kenyon, S. J., Kolotilov, E. A., Ibragimov, M. A., \& Mattei, J. A. 2000, ApJ, 531,1028

Kenyon, S. J., \& Hartmann, L. W. 1991, ApJ, 383, 664

Kospal, A., Abraham, P., \& Acosta-Pulido, J. 2005, IBVS, 5661

Kospal, A., Abraham, P., Prusti, T., et al. 2006, ASPC, 349, 269

Lada, \& Wilking 1984, ApJ, 287, 610

Leinert, Ch., Graser, U., Przygodda, F., et al. 2003, Ap\&SS, 285, 73

Lin, B. D. C., \& Papaloizou, J. C. B. 1985, Protostars and Planets II

Magnier, E. A., Waters, L. B. F. M., Groot, P. J., et al. 1999, A\&A, 346, 441

Mallas, J. H., \& Kreimer, E. 1970, The Messier Album

McGehee, P. M., Smith, J. A., Henden, A. A., et al. 2004, ApJ, 616, 1058

McNeil, J. W. 2004, IAU Circ., 8284

Megeath, S. T., Allen, L. E., Allgaier, E., et al. 2006, IAUS, 237, 167

Muzerolle, J., Megeath, S. T., Flaherty, K. M., et al. 2005, ApJ, 620, L107

Ojha, D. K., Ghosh, S. K., Tej, A., et al. 2006, MNRAS, 368, 825

Reipurth, B., \& Aspin, C. 2004, ApJ, 606, L119

Rettig, T. W., Brittain, S. D., Gibb, E. L., et al. 2005, ApJ, 626, 245

Rossi, C., Errico, L., Friedjung, M., et al. 1999, A\&AS, 136, 95

Sandell, G., \& Weintraub, D. A. 2001, ApJ, 134, 115

Shevchenko 1999, Astron. Rep., 43, 246

Semkov, E. H. 2004, IBVS, 5578

Semkov, E. H. 2006, IBVS, 5683

Smith, J., Tucker, D. L., Kent, S., et al. 2002, AJ, 123, 2121

Stetson, P. 2000, PASP, 112, 925

Tsukagoshi, T., Kitamura, Y., Kawabe, R., et al. 2005, PASJ, 57, L21

Vacca, W. D., Cushing, M. C., \& Simon, T. 2004, ApJ, 609, L29

Van den Ancker, M. E., Blondel, P. F. C., Tjin A Djie, H. R. E., et al. 2004, MNRAS, 349, 1516

Van der Bliek, N. S., et al. 1996, A\&AS, 109, 547

Walter, F. M., Stringfellow, G. S., Sherry, W. H., et al. 2004, AJ, 128, 1872 